\title{
Electrochemical generation of surface enhanced Raman scattering substrates for the determination of folic acid
}

\author{
W. Cheuquepan*, S. Hernandez, M. Perez-Estebanez, L. Romay, A. Heras, A. Colina* \\ Department of Chemistry, Universidad de Burgos, Pza. Misael Bañuelos s/n, E-09001 Burgos, Spain
}

\section{A R T I C L E I N F O}

\section{Keywords:}

Spectroelectrochemistry

Surface enhanced Raman Scattering

SERS

RAMAN

\begin{abstract}
A B S T R A C T
Electrochemistry is a very useful technique to generate surface enhanced Raman scattering (SERS) substrates. SERS depends on two mechanisms: the electromagnetic effect, related to the plasmonic properties of the nanostructures in the substrate, and the chemical mechanism, related to the interaction between the target molecule and the substrate. Thus, Raman enhancement for a molecule strongly depends on the nature of the nanostructures that form the substrate. In this work, time-resolved Raman spectroelectrochemistry is used to generate SERS substrates, obtaining the Raman signal during the generation of the substrate, which is very useful to obtain valuable information on the Raman performance of the substrate. Two metals, Au and Ag, have been used to determine folic acid in a drug. In this example, Au SERS substrates are highly influenced by interfering compounds present in the drug tables. On the contrary, Ag SERS substrates have been demonstrated to yield very good figures of merit in the determination of folic acid in this complex sample.
\end{abstract}

\section{Introduction}

Surface Enhanced Raman Scattering (SERS) is a surface process that is widely used for analysis [1-4]. This phenomenon was discovered during the electrochemical roughening of a silver electrode in the study of pyridine [5]. Since then, a number of methods have been developed to prepare SERS substrates [6-9]. Most sensitive substrates are usually prepared by physical or chemical synthesis of nanostructures deposited on a surface to detect a target molecule [6-9]. However, the reproducibility of substrates for quantitative analysis remains a challenge $[4,6,10]$. The high sensitivity provided by this methods is mainly due to the generation of hot spots on the substrate $[4,6,8]$, which shows a low reproducibility because it is not easy to reproduce these hot spots. Moreover, target molecules cannot be easily removed from the SERS substrates because of the high adsorption of the target molecule on the plasmonic nanostructures. Therefore, a different SERS substrate should be used for each sample. Electrochemistry is an alternative for preparing SERS substrates in a very simple and fast way $[7,10]$. For years, different protocols have been developed for roughening electrodes [7], most of them yielding useful SERS substrates used to study electrode processes [11]. However, this methodology has been scarcely used for quantitative analysis because of the difficulty in obtaining reproducible SERS substrates. Nevertheless, a new methodology based on the use of screen-printed electrodes has been developed during the last years $[10,12,13]$. SERS substrates based on SPEs can be prepared in a reproducible way [10,12-14], obtaining an excellent sensitivity [15]. Moreover, the use of time resolved Raman spectroelectrochemistry (TR-Raman-SEC) [16] helps to follow the Raman signal during all the substrate preparation process, obtaining continuous information on the signal enhancement during the SERS substrate electrogeneration $[17,18]$. Electrochemistry can generate in-situ SERS substrates, avoiding tedious operations to prepare the nanostructured substrate and modify the electrode. Raman-SEC is particularly interesting, since it allows us to take advantage of electrochemical surface enhanced Raman scattering (EC-SERS) and other phenomena like electrochemical surface oxidation enhanced Raman scattering (EC-SOERS), which have been successfully used for analysis $[10,13,19]$. It is noteworthy the capability of EC-SERS and EC-SOERS to perform determinations of molecules in complex matrices [20] thanks to the spectroscopic fingerprint provided by Raman spectroscopy. Moreover, electrochemistry is very useful for SERS analysis because it can be used to adsorb the target molecule on the electrode surface [21-24], increasing the Raman signal.

Mainly, two metals, Au and Ag, are used to prepare SERS substrates because of the plasmonic properties of the generated nanostructures, which help to enhance the Raman signal. The Raman response of a target molecule on nanostructures of these metals can yield different behavior [6]. Two different mechanisms are responsible for the SERS

\footnotetext{
* Corresponding author.

E-mail addresses: wcheuquepan@ubu.es (W. Cheuquepan), acolina@ubu.es (A. Colina).
} 
effect. On the one hand, the electromagnetic effect enhances the Raman signal because of the electromagnetic fields generated by the excitation of localized surface plasmons of the substrate $[6,25]$. On the other hand, the chemical effect enhances the Raman signal due to the interaction of the molecule with the substrate $[6,25]$. This interaction strongly depends on the chemical structure of the molecule and the charge transfer between the molecule and the surface of the substrate. Thus, different substrates yield different enhancement factors for the same molecule. TR-Raman-SEC allows us to obtain information on the interaction of the target molecule with the SERS substrate in a simple and fast way. In this work, we have selected folic acid as the target molecule, studying the SERS response of this analyte on gold and silver substrates.

Folic acid (FA), also known as vitamin B9 or pteroyl-L-glutamic acid or folate in its anionic form, is a water-soluble vitamin that belongs to the B complex family with a significant biological interest in different metabolic pathways and processes [26]. Its chemical structure is divided into three parts: a pterin linked by a methylene bridge to $p$ aminobenzoic acid joined by a peptide linkage to a molecule of the amino acid L-glutamic acid [26].

FA presents a low solubility in water $\left(1.6 \mathrm{mg} / \mathrm{L}\right.$ at $\left.25^{\circ} \mathrm{C}\right)$ and exhibits self-aggregation in a wide $\mathrm{pH}$ range. The solubility in water is slightly increased in acidic or basic medium, being more stable in alkaline medium [26].

Folic acid plays a vital role in numerous biochemical processes, such as the formation and maturation of blood cells and cell multiplication. With vitamin B12, FA in its form 5,6,7,8-tetrahydrofolate or 5,6,7,8-tetrahydropteroylmonoglutamic acid (THF) participates in the regulation of genetic activity, in the synthesis of purines and pyrimidines, in the metabolism of amino acids, such as homocysteine, and in the biosynthesis of DNA and RNA. Furthermore, FA is necessary for the normal development of tissues, mainly in those associated with rapid cell division (embryos) [26].

In this way, pregnant women must maintain the concentration of FA at appropriate levels, since the deficiency of this vitamin can cause neural tube defects in the fetus, such as spina bifida or anencephaly, in addition to complications during pregnancy. Other harmful health effects of FA deficiency are megaloblastic anemia, coronary heart disease, certain types of cancer, neurological disorders, fertility problems, etc [26].

Numerous analytical methods have been proposed for the determination of FA in fortified foods as well as in natural sources or pharmaceutical drugs such as UV-Vis spectrophotometry [27,28], HPLC [29], HPLC-MS [30] or chemiluminescence [31]. However, these techniques usually require tedious sample pretreatments, which increase the analysis time.

For this reason, electrochemical techniques have gained importance, thanks to the speed of measurement and their low cost. Thus, the most widely used electrochemical methods for the determination of folic acid are based on the use of modified electrodes [32-35].

FA has also been determined using SERS, achieving very good limits of detection. For example, wafer-scale SERS metallic nanotube arrays have provided very sensitive Raman responses [36], polyamide@Ag coralloid nanoarrays with 3D high-density hot spots have been used for ultrasensitive detection [37], large-area gold-capped silicon nanopillars have also provided good sensitivity [38] or graphene oxide/Ag nanoparticle hybrids have been also used [39]. All these methods are based on the preparation of complex SERS substrates requiring long and tedious substrate preparations.

Both electrochemistry and SERS have been used to determine FA, but, to the best of our knowledge, TR-Raman-SEC has not ever been used to quantify FA.

In this work, TR-Raman SEC has been used for studying the electrochemical generation of SERS substrates of $\mathrm{Au}$ and $\mathrm{Ag}$, to obtain the best conditions to determine FA in a commercial drug using a very simple protocol that can be used in any laboratory.

\section{Experimental}

\subsection{Chemicals and materials}

Folic acid (97\%, Sigma-Aldrich), $\mathrm{HClO}_{4}$, (60\%, Sigma-Aldrich), $\mathrm{KCl},\left(99+\%\right.$, ACROS Organics), NaOH (ACROS Organics), $\mathrm{LiClO}_{4}$, (99+\%, ACROS Organics) were analytical grade and used as received without further purification. Yodocefol@ (ITALFARMACO), each capsule contains: $400 \mu \mathrm{g}$ of folic acid, $262 \mu \mathrm{g}$ of potassium iodide, $2 \mu \mathrm{g}$ de vitamin B12, and the following excipients: 110 mesh lactose monohydrate, microcrystalline cellulose, sodium potato starch glycolate, calcium stearate, trisodium citrate, citric acid and maltodextrins. For safety considerations, all handling and processing were carefully performed during all experiments. Aqueous solutions were freshly prepared using ultrapure water $\left(18.2 \mathrm{M} \Omega \mathrm{cm}\right.$ resistivity at $25{ }^{\circ} \mathrm{C}, 2 \mathrm{ppb}$ TOC, Milli-Q Direct 8, Millipore).

\subsection{Instrumentation}

Raman measurements were performed with a customized SPELEC RAMAN (Metrohm-DropSens) and a customized RAMANCELL (Metrohm-DropSens). A $785 \mathrm{~nm}$ laser source was used, fixing the laser power at $102 \mathrm{~mW}\left(325 \mathrm{~W} \mathrm{~cm}^{-2}\right)$ in all experiments. Silver DRP-010 and gold DPR-220BT screen-printed electrodes (SPEs) (MetrohmDropSens) were used for Raman SEC measurements (SPE-WE diameter $=4 \mathrm{~mm}$ ). SPELEC RAMAN instrument is controlled by DropView SPELEC software (Metrohm-DropSens), performing simultaneous, time-resolved SEC experiments with fully synchronized data acquisition. A different SPE has been used for each Raman-SEC experiment.

\section{Results and discussion}

Raman-SEC is considered a poor technique for analysis because of the low sensitivity of Raman spectroscopy. However, the Raman signal can be significantly enhanced using appropriate substrates. Two different strategies are usually employed to enhance the Raman signal during an electrochemical experiment: EC-SERS [13,14,21] and EC-SOERS $[12,19,20,40]$. In the case of folic acid, EC-SERS provides the best response. Two different substrates, gold and silver, were investigated to obtain a sensitive, reproducible and robust response.

For the two metals, experimental conditions were optimized to obtain a good Raman response. Raman spectra were obtained using an integration time of $1 \mathrm{~s}$ with a laser power of $102 \mathrm{~mW}$. This power can be considered high with respect to values found in the literature. However, the spot size in our experimental setup is much bigger, $200 \mu \mathrm{m}$, than the usual spot size used in confocal Raman microscopy, around $2 \mu \mathrm{m}$. Thus, in our case the power per unit area was $325 \mathrm{~W} \cdot \mathrm{cm}^{-2}$.

Initially, Raman experiments were performed to obtain the Raman spectra of a FA solid sample and a $0.05 \mathrm{M}$ FA in $0.1 \mathrm{M} \mathrm{NaOH}$. Fig. 1 shows the Raman spectra for the FA solid and FA in solution. As can be seen, the two samples show similar and characteristic bands. It should be noted that the spectrum in solution is multiplied 5 times for a better comparison (blue line in Fig. 1).

Table 1 shows the assignation of the Raman bands observed in Fig. 1 for the different vibration modes of FA [41]. FA Raman bands in solution are slightly shifted with respect to FA solid because of the variation of the physical-chemical environment of the molecules. FA molecules are solvated, having different surroundings respect to the solid sample.

SERS substrates were electrochemically generated using cyclic voltammetry, scanning the potential to generate ions of the metal in the solution that are complexed or precipitate on the electrode surface. These ions were then reduced to generate plasmonic structures of these metals that enhance the Raman signal. The electrochemical 


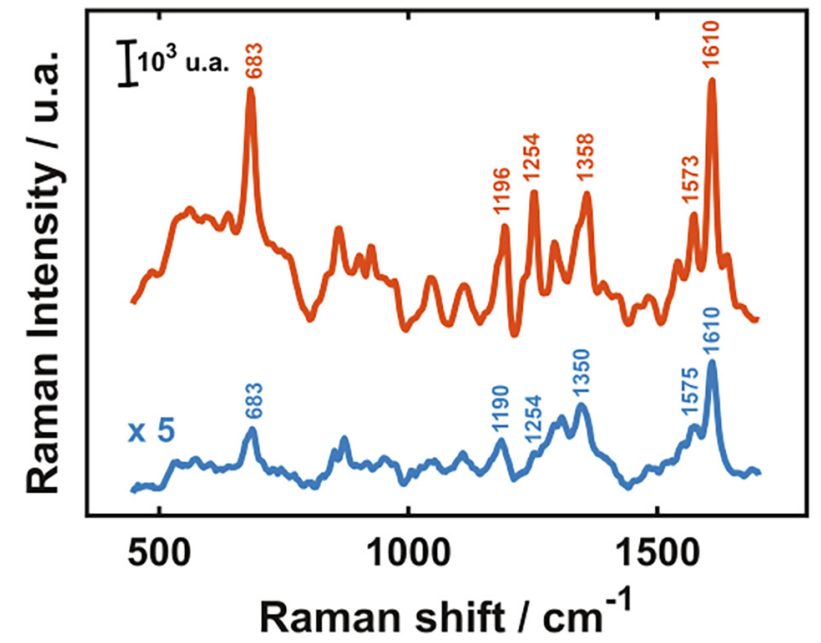

Fig. 1. Raman spectra of FA solid (orange line) and FA in solution (blue line, $0.05 \mathrm{M}$ ). For a better comparison, the spectrum in solution (blue line) has been multiplied five times.

Table 1

Raman band assignment for FA found in literature [41].

\begin{tabular}{|c|c|c|}
\hline $\begin{array}{l}\text { Solid Raman bands / } \\
\mathrm{cm}^{-1}\end{array}$ & $\begin{array}{l}0.05 \mathrm{M} \text { solution bands / } \\
\mathrm{cm}^{-1}\end{array}$ & Band assignment \\
\hline 683 & 683 & $\nu(\mathrm{CC}) \mathrm{PHEN}$ \\
\hline 1196 & 1190 & $\begin{array}{l}\nu\left(\mathrm{C}-\mathrm{NH}_{2}\right)+\delta\left(\mathrm{NH}_{2}\right)+\nu(\mathrm{CC}, \\
\mathrm{CN}) \mathrm{pt}\end{array}$ \\
\hline 1254 & 1254 & $\nu(\mathrm{CC}, \mathrm{CN}, \mathrm{CO}) \mathrm{pt}$ \\
\hline 1358 & 1350 & $\delta(\mathrm{OH}) \mathrm{pt}+\delta\left(\mathrm{CH}_{2}\right) \mathrm{glu}$ \\
\hline 1573 & 1575 & $\begin{array}{l}\delta(\mathrm{OH}) \mathrm{pt}+\delta\left(\mathrm{CH}_{2}\right) \\
\mathrm{GLU}+\delta(\mathrm{OH}) \mathrm{glu}\end{array}$ \\
\hline 1610 & 1610 & $\delta(\mathrm{CC}, \mathrm{CN}) \mathrm{pt}, \mathrm{oop}$ \\
\hline
\end{tabular}

$\nu=$ stretching; $\delta=$ bending; oop $=$ out of plane; $\mathrm{pt}=$ peteridine, $\mathrm{phen}=$ phenilen, glu = glutamic acid.

protocol depends on the metal. Three different electrolytic media were investigated, $0.1 \mathrm{M} \mathrm{KCl}, 0.1 \mathrm{M} \mathrm{NaOH}$ and $0.1 \mathrm{M} \mathrm{HClO}_{4}+5 \mathrm{mM} \mathrm{KCl}$, with the best results being obtained for $0.1 \mathrm{M} \mathrm{KCl}$ in the case of AuSPEs and $0.1 \mathrm{M} \mathrm{HClO}_{4}+5 \mathrm{mM} \mathrm{KCl}$ for Ag-SPEs.

For Au-SPEs, the CV starts at $+0.70 \mathrm{~V}$ in anodic direction, scanning the potential between $-0.10 \mathrm{~V}$ and $+1.20 \mathrm{~V}$ at $0.02 \mathrm{~V} \mathrm{~s}^{-1}$ in a $10 \mu \mathrm{M}$
FA, $0.1 \mathrm{M} \mathrm{KCl}$ solution. Fig. 2 shows the $\mathrm{CV}$ together to the voltaRamangram (evolution of a Raman band respect to the applied potential) at $1568 \mathrm{~cm}^{-1}$ and the Raman spectrum at $-0.10 \mathrm{~V}$ in the cathodic scan, see the asterisk in Fig. 2a.

The CV shows that the Au SPE is oxidized at $+0.90 \mathrm{~V}$, generating $\mathrm{Au}(\mathrm{III})$ in solution, which is complexed in presence of chloride. As can be observed, the SERS signal at $1568 \mathrm{~cm}^{-1}$ increases when the $\mathrm{AuCl}_{4}^{-}$ is reduced to $\mathrm{Au}(0)$. Under similar conditions, $\mathrm{Au}$ nanoparticles have been observed in previous works [14], which should be the nanostructures responsible of the Raman signal enhancement. In this case, the Raman band is slightly shifted to a lower Raman shift. The Raman signal increases up to $+0.25 \mathrm{~V}$, reaching a constant value during the rest of the cathodic scan. The Raman spectrum is well defined, Fig. $2 b$, being suitable for quantification. A tentative calibration of FA was performed using only three concentrations from 3 to $7.5 \mu \mathrm{M}$, Fig. 3 .

As can be observed, a good linear response is obtained $\left(\mathrm{R}^{2}=0.99\right)$. A Yodocefol ${ }^{\circledR}$ sample was prepared, dissolving a tablet in $25 \mathrm{~mL}$ of $0.1 \mathrm{M} \mathrm{NaOH}$ solution, a medium in which folic acid is known to be soluble as folate, but a dispersion is obtained. As has been indicated in the experimental section the tablet contains potentially interfering compounds. Some of the tablet components are not soluble in this medium, so a simple pre-treatment of the sample prior to its quantification was performed. The obtained dispersion was decanted in a first step. Next, $1 \mathrm{~mL}$ aliquots were placed in Eppendorf vials and centrifuged at $4000 \mathrm{rpm}$ for $28 \mathrm{~min}$. The supernatant was taken as drug sample to be quantified. A dilution, neutralizing the basic solution and adding the appropriate electrolytic medium, was performed. A theoretically $4.5 \mu \mathrm{M}$ FA from the Yodocefol ${ }^{\circledR}$ sample in $0.1 \mathrm{M} \mathrm{KCl}$ was prepared. The green point in Fig. 3 corresponds to the test sample prepared from the Yodocefol ${ }^{\circledR}$ tablet. As can be observed, the Raman signal obtained for this sample is much lower than the expected one from the calibration data. The concentration would be underestimated using this method. Therefore, Au-SPEs are not useful for the quantification of FA in this complex sample. Actually, electrochemistry provides very valuable information on the limited performance of the Au-SPEs. Fig. 4 shows a comparison of two cyclic voltammetry experiments performed for a sample containing $5 \mu \mathrm{M}$ FA (orange line) and a Yodocefol sample (blue line) in $0.1 \mathrm{KCl}$. As can be observed, the pharmaceutical sample shows a clear much lower oxidation current of the gold surface that exhibits a great influence on the generation of nanostructures responsible of the SERS signal. During the oxidation of the gold electrode, some of the different compounds contained in the tablets interact with the gold surface, being responsible of the decrease of both the voltammetric and the Raman signal. (a)

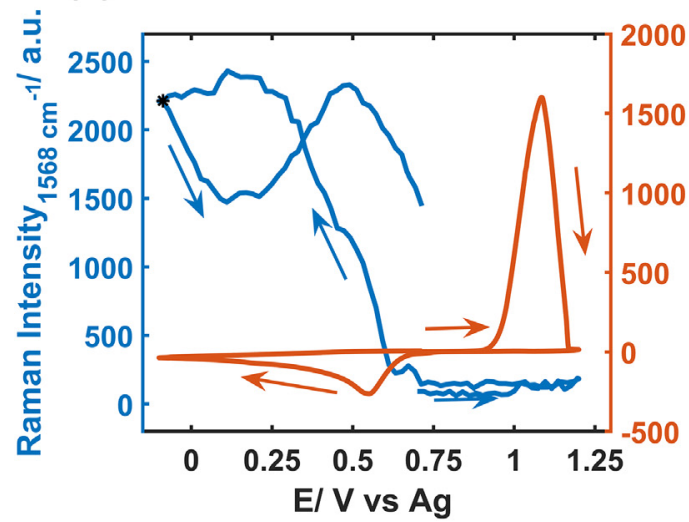

(b)

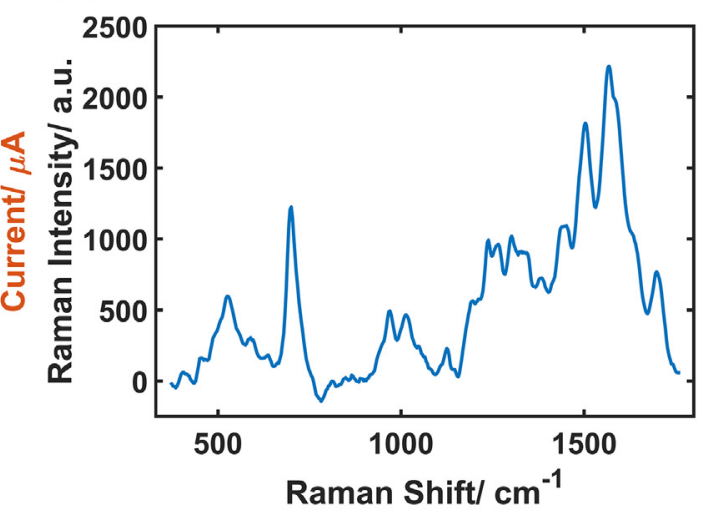

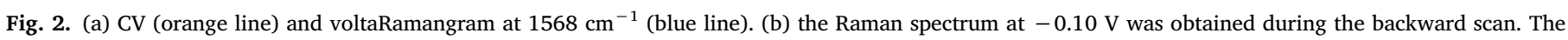

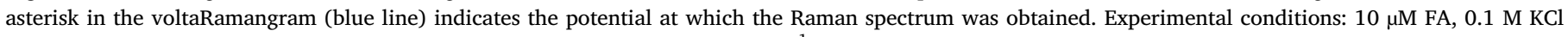
solution. The potential was scanned between $-0.10 \mathrm{~V}$ and $+1.20 \mathrm{~V}$ at $0.02 \mathrm{Vs}^{-1}$, starting at $+0.70 \mathrm{~V}$ in anodic direction using a Au-SPE. 


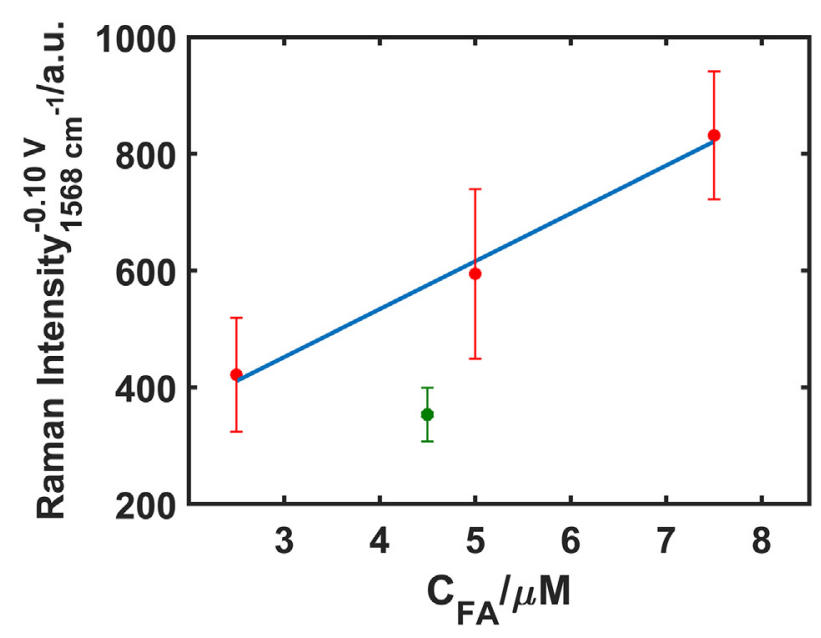

Fig. 3. Calibration curve of FA obtained from the voltaRamangrams at the vertex potential, $-0.10 \mathrm{~V}$, at $1568 \mathrm{~cm}^{-1}$. The green point in the figure corresponds to the theoretical concentration value of a Yodocefol ${ }^{\circledR}$ sample. Experimental conditions in the caption of Fig. 2. Each point was replicated three times.

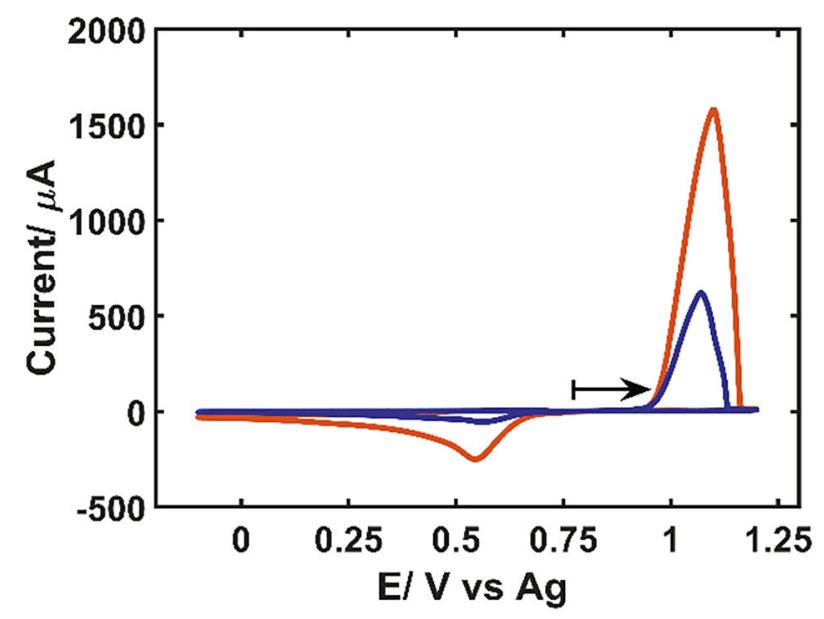

Fig. 4. CVs recorded for using a $5 \mu \mathrm{M}$ FA solution (orange line) and a Yodocefol sample (blue line) in $0.1 \mathrm{KCl}$ using a Au-SPE. Experimental conditions in the caption of Fig. 2.

This unexpected process in the test sample shows an important influence of the compounds accompanying FA in the tablet in the generation of the SERS substrate. The enhancement of the Raman signal decreases, being impossible to obtain a reliable Raman spectrum to determine FA in the drug tablet.

Results on Ag-SPEs using a $0.1 \mathrm{M} \mathrm{HClO}_{4}$ and $5 \mathrm{mM} \mathrm{KCl}$ medium yielded the best SERS response. In all cases, a much better sensitivity, but also reproducibility, of the Raman signal was achieved using Ag SERS substrates as will be shown below.

The optimal conditions of electrosynthesis for a suitable SERS substrate on the Ag-SPE were obtained using CV, starting at $+0.40 \mathrm{~V}$ in cathodic direction and scanning the potential between $+0.40 \mathrm{~V}$ and $-0.40 \mathrm{~V}$ at $0.02 \mathrm{~V} \mathrm{~s}^{-1}$ in a $5 \mathrm{mM} \mathrm{KCl}$ and $0.1 \mathrm{M} \mathrm{HClO}_{4}$ solution. This electrochemical protocol was selected because an excellent reproducibility was obtained under these conditions. Fig. 5a shows the Raman-SEC signals obtained during this experiment. CV shows a high anodic current at the starting potential, $+0.40 \mathrm{~V}$, because at this potential the oxidation of the silver surface $\left(\mathrm{Ag}^{0}\right)$ is taking place, yielding both silver chloride $(\mathrm{AgCl})$ and silver ions $\mathrm{Ag}(\mathrm{I})$. $\mathrm{At}+0.30 \mathrm{~V}$, a first reduction peak can be observed due to the reduction of $\mathrm{Ag}(\mathrm{I})$ to $\mathrm{Ag}(0)$ which is deposited on the surface. A second reduction at $0.00 \mathrm{~V}$ transforms $\mathrm{AgCl}$ in $\mathrm{Ag}(0)$, which is also deposited on the surface of the working electrode. During the backward scan two oxidation peaks are observed. The first one at $-0.10 \mathrm{~V}$ is not related to any process in which silver is involved. Moreover, this peak is also observed starting the $\mathrm{CV}$ at $-0.40 \mathrm{~V}$ in a $5 \mathrm{mM} \mathrm{KCl}$ and $0.1 \mathrm{M} \mathrm{HClO}_{4}$ solution without the test molecule and without oxidizing the Ag SPE (data not shown). Therefore, this peak must be related to some compound of the Ag-SPE ink. The second anodic peak observed at $+0.10 \mathrm{~V}$ is related to the $\mathrm{Ag}(0)$ oxidation to generate $\mathrm{AgCl}$. Finally, a high anodic current due to the oxidation of the silver electrode to generate $\mathrm{Ag}(\mathrm{I})$ is observed from $+0.30 \mathrm{~V}$ forwards.

The electrochemical processes taking place at the Ag electrode surface can be related to the Raman signal obtained during this SEC experiment. The Raman spectrum of FA at $-0.20 \mathrm{~V}$ in the backward scan is shown in Fig. 5b. Fig. 5a also shows the voltaRamangram at $1582 \mathrm{~cm}^{-1}$. A linear baseline between $1552 \mathrm{~cm}^{-1}$ and $1597 \mathrm{~cm}^{-1}$ has been used to obtain the voltaRamangram response for quantitative purposes, removing the background. This baseline will be used to calculate the Raman intensity of the different standards in the calibration for quantitative purposes. As can be observed in the voltaRamangram at $1582 \mathrm{~cm}^{-1}$ (blue line in Fig. 5a), Raman intensity increases during the reduction of $\mathrm{AgCl}$, from $0.00 \mathrm{~V}$ to $-0.10 \mathrm{~V}$ in the forward scan. The enhancement of the Raman signal can be ascribed to the generation of Ag nanoparticles, as has been observed under similar conditions in previous works [19]. Next, Raman intensity remains constant up to the vertex potential $(-0.40 \mathrm{~V})$. When the scan direction is changed, the Raman intensity increases up to $-0.20 \mathrm{~V}$ in the backward scan, where it reaches its maximum (black asterisk in the voltaRamangram, Fig. 5a). This increment of the Raman intensity after changing the scan direction could be related to changes in the double layer that favours the adsorption of FA on the Ag nanostructures. During the oxidation of the Ag surface in the backward scan, the Raman signal completely decreases at the second oxidation peak at $+0.10 \mathrm{~V}$, reaching a zero value because all the nanostructures responsible of the enhancement of the Raman signal have been oxidized.

This analytical protocol described in Fig. 5 can be used to determine FA in solution at low concentrations. Thus, a calibration set of known FA concentration samples between 1 and $10 \mu \mathrm{M}$ was prepared, with standard solutions of $1,2,4,6,8$, and $10 \mu \mathrm{M}$ of FA in the electrolytic medium indicated above. Fig. 6 a shows the voltaRamangrams for three concentration samples, evidencing that the higher is the concentration, the higher is the recorded Raman intensity. Fig. 6b shows the calibration curves obtained for this concentration range at two Raman bands ascribed specifically to FA: 710 and $1582 \mathrm{~cm}^{-1}$. Green points in Fig. $6 \mathrm{~b}$ corresponds to the prediction of a Yodocefol® ${ }^{\circledR}$ test sample.

Table 2 shows the figures of merit of the two linear regression models constructed with Raman intensity values at 710 and $1582 \mathrm{~cm}^{-1}$. The two regression lines show very good linearity and very good repeatability of the slope $(\% \mathrm{RSD}=7.2, \mathrm{n}=4)$ for EC-SERS measurements. The LOD was 0.76 and $0.72 \mu \mathrm{M}$, for the calibration at 710 and $1582 \mathrm{~cm}^{-1}$, respectively, indicating the high sensitivity of ECSERS. The LOD was calculated using the standard deviation $\left(S_{y x}\right)$ and the slope $(k)$ of the calibration curve as $3 S_{y x} / k$ [42]. Therefore, these good figures of merit indicate that this method can be used to quantify test samples. The two Raman shifts provide similar analytical methods, but $1582 \mathrm{~cm}^{-1}$ exhibits the best sensitivity. The capability to measure at very different Raman shifts is another important advantage of EC-SERS in analysis, because the determination of a molecule can be performed in different zones of the spectrum if the signal of another molecule is interfering with some peaks of our analyte.

Once demonstrated that this Raman SEC method is reliable, repeatable and sensitive, the determination of FA in a drug (Yodocefol $\AA$ ) was performed, probing the capability of prediction of the method for real 
(a)

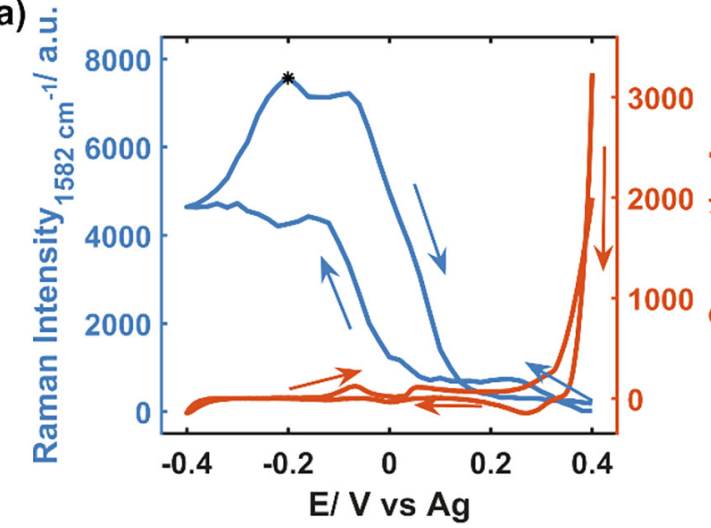

(b)

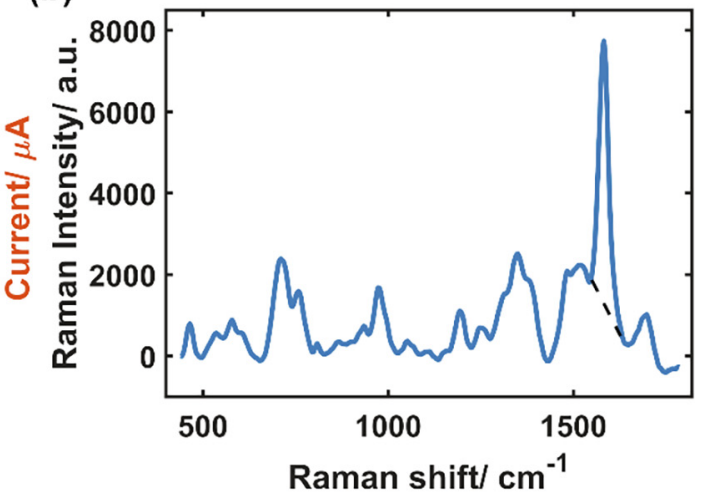

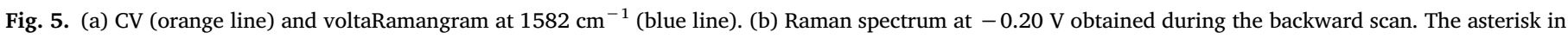

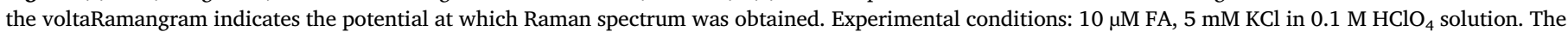
potential scan was performed from $+0.40 \mathrm{~V}$ to $-0.40 \mathrm{~V}$ at $0.02 \mathrm{~V} \mathrm{~s}^{-1}$ using a Ag-SPE.

(a)

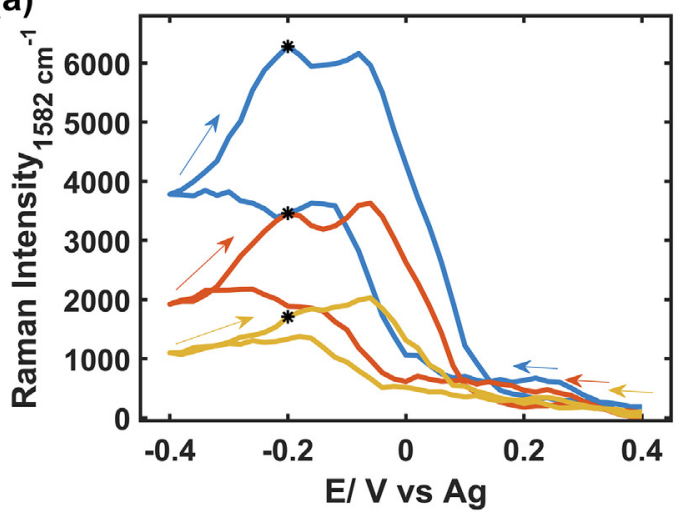

(b)

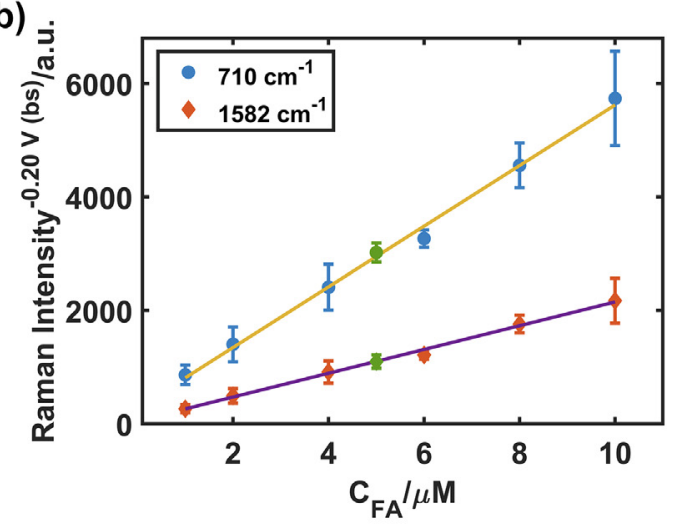

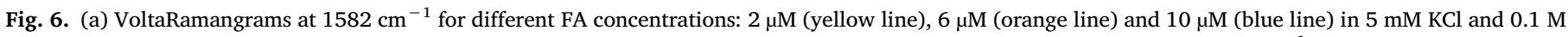

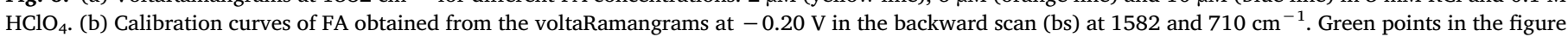

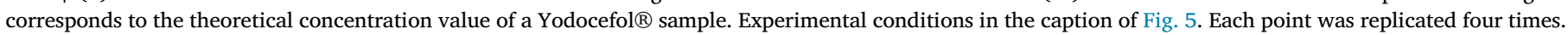
We have modified y label in Fig 6.b

Table 2

Figures of merit for the linear regression models obtained from the raman intensity values at 710 and $1582 \mathrm{~cm}^{-1}$, at $-0.20 \mathrm{~V}$ in the backward scan.

\begin{tabular}{|c|c|c|c|c|c|}
\hline Raman shift $\left(\mathrm{cm}^{-1}\right)$ & Slope $\left(\mu \mathrm{M}^{-1}\right)$ & Intercept (a.u.) & $\mathrm{R}^{2}$ & $\mathrm{~S}_{\mathrm{yx}}$ & $\mathrm{LOD}(\mu \mathrm{M})$ \\
\hline 710 & 209.65 & 53.9 & 0.996 & 52.90 & 0.76 \\
\hline 1582 & 534.42 & 278.11 & 0.996 & 129.51 & 0.73 \\
\hline
\end{tabular}

$\mathrm{R}^{2}$ : coefficient of determination; $\mathrm{S}_{\mathrm{yx}}$ : standard deviation of residuals; LOD: limit of detection.

samples. Yodocefol ${ }^{\circledR}$ tablet contains $262 \mu \mathrm{g}$ of KI (equivalent to $200 \mu \mathrm{g}$ of $\left.\mathrm{I}_{2}\right), 400 \mu \mathrm{g}$ of FA and $2 \mu \mathrm{g}$ of vitamin $\mathrm{B} 12$ and other compounds in unknown concentration as is indicated in the experimental section. Therefore, the analyte is accompanied by potential interfering compounds as has been demonstrated for Au-SPEs. Following the simple protocol shown above, the tablet was dissolved in $25 \mathrm{~mL}$ of $0.1 \mathrm{M}$ $\mathrm{NaOH}$ solution, obtaining a dispersion that is decanted. Next, $1 \mathrm{~mL}$ aliquots were placed in Eppendorf vials and centrifuged at $4000 \mathrm{rpm}$ for $28 \mathrm{~min}$. The supernatant was taken as a drug sample to be quantified. Finally, the basic solution was neutralized with $\mathrm{HClO}_{4}$ and a theoretically $5 \mu \mathrm{M}$ FA from the Yodocefol ${ }^{\circledR}$ sample in $0.1 \mathrm{M} \mathrm{HClO}_{4}$ and $5 \mathrm{mM}$ $\mathrm{KCl}$ was prepared. In the case of the Ag-SPEs, a robust SERS substrate was obtained, obtaining an estimated concentration of $5.0 \pm 0.4 \mu \mathrm{M}$, demonstrating that the new methodology is not affected by the interfering compounds contained in the drug tablet. Therefore, the esti- mated amount of FA in the Yodocefol ${ }^{\circledR}$ tablet was $401.6 \pm 3.2 \mu \mathrm{g}$, which is very close to the amount of FA contained in the drug tablet. Moreover, a remarkable low dispersion of the results was obtained. This value demonstrates the good performance of the EC-SERS method based on the oxidation/reduction of a silver SPE in a $5 \mathrm{mM} \mathrm{KCl}$ and $0.1 \mathrm{M} \mathrm{HClO}_{4}$ medium for the determination of FA.

\section{Conclusions}

TR-Raman-SEC has been demonstrated to be an excellent analytical technique for the determination of FA. It is noteworthy, that electrochemistry is the motor of the reaction used to quantify the analyte using spectroscopy. In TR-Raman-SEC, electrochemistry is used to generate plasmonic nanostructures that enhance the optical response of the target molecule. Therefore, the excellent union of electrochemistry 
and optical techniques facilitates the development of analytical methods. Two different metals have been used to develop a suitable SERS substrate for the determination of FA. SERS is highly influenced by the interaction between the molecule and the plasmonic substrate, obtaining very different results with different metal electrodes. Ag SPEs have provided better results in terms of sensitivity for FA than Au SPEs. The new TR-Raman-SEC method based on the oxidation/reduction of an Ag SPE has been successfully used to determine FA in Yodocefol ${ }^{\circledR}$, a commercial drug that contains potential interfering compounds, demonstrating the robustness of these techniques for complex samples. Particularly, Au SPEs are strongly influenced by the interfering compounds of Yodocefol ${ }^{\circledR}$. It is noteworthy that a very simple sample pretreatment of the sample prior to its quantification is required. Quantification of test samples can be performed using a univariate calibration, demonstrating that different spectral bands can be used for quantification. SEC techniques can be easily implemented for multivariate analysis, but in many cases, a simple univariate analysis is enough to obtain high quality analytical information. Additionally, TR-Raman-SEC provides a fingerprint of the molecule, providing unequivocally information on the compound that is being determined.

\section{Author contribution}

S.H, L.R, M.P.E and W.Ch. contributed to the acquisition of data. W. Ch, S.H, M.P.E, A.H and A.C. contributed to the conception, design and implementation of the research and to the analysis and interpretation of the results. All the authors contributed to the writing of the manuscript and to the revision of the manuscript critically for important intellectual content. This work has been headed by W. Ch. and A.C.

\section{Declaration of Competing Interest}

The authors declare that they have no known competing financial interests or personal relationships that could have appeared to influence the work reported in this paper.

\section{Acknowledgements}

Authors acknowledge the financial support from Ministerio de Economía, y Competitividad (Grant CTQ2017-83935-R-AEI/FEDERUE), Junta de Castilla y León (Grant BU297P18) and Ministerio de Ciencia, Innovación y Universidades (Grant RED2018-102412-T). W. Ch. thanks JCyL for his postdoctoral fellowship (Grant BU297P18). S.H. and M.P-E. thanks JCyL and European Social Fund for their predoctoral fellowships.

\section{References}

[1] C. Zong, M. Xu, L.-J. Xu, T. Wei, X. Ma, X.-S. Zheng, R. Hu, B. Ren, Surfaceenhanced Raman spectroscopy for bioanalysis: reliability and challenges, Chem. Rev. 118 (10) (2018) 4946-4980, https://doi.org/10.1021/acs.chemrev.7b00668.

[2] R. Gillibert, J.Q. Huang, Y. Zhang, W.L. Fu, M. Lamy de la Chapelle, Explosive detection by Surface Enhanced Raman Scattering, TrAC - Trends Anal. Chem. 105 (2018) 166-172, https://doi.org/10.1016/j.trac.2018.03.018.

[3] R. Goodacre, D. Graham, K. Faulds, Recent developments in quantitative SERS: moving towards absolute quantification, TrAC - Trends Anal. Chem. 102 (2018) 359-368, https://doi.org/10.1016/j.trac.2018.03.005.

[4] S.E.J. Bell, G. Charron, E. Cortés, J. Kneipp, M.L. Chapelle, J. Langer, M. Procházka, V.i. Tran, S. Schlücker, Towards reliable and quantitative surfaceenhanced Raman scattering (SERS): from key parameters to good analytical practice, Angew. Chemie - Int. Ed. 59 (14) (2020) 5454-5462, https://doi.org/ 10.1002/anie.v59.1410.1002/anie.201908154.

[5] M. Fleischmann, P.J. Hendra, A.J. McQuillan, Raman spectra of pyridine adsorbed at a silver electrode, Chem. Phys. Lett. 26 (2) (1974) 163-166, https://doi.org/ 10.1016/0009-2614(74)85388-1.

[6] J. Langer, D. Jimenez de Aberasturi, J. Aizpurua, R.A. Alvarez-Puebla, B. Auguié, J. J. Baumberg, G.C. Bazan, S.E.J. Bell, A. Boisen, A.G. Brolo, J. Choo, D. Cialla-May, V. Deckert, L. Fabris, K. Faulds, F.J. García de Abajo, R. Goodacre, D. Graham, A.J. Haes, C.L. Haynes, C. Huck, T. Itoh, M. Käll, J. Kneipp, N.A. Kotov, H. Kuang, E.C. Le Ru, H.K. Lee, J.-F. Li, X.Y. Ling, S.A. Maier, T. Mayerhöfer, M. Moskovits, K. Murakoshi, J.-M. Nam, S. Nie, Y. Ozaki, I. Pastoriza-Santos, J. Perez-Juste, J. Popp,
A. Pucci, S. Reich, B. Ren, G.C. Schatz, T. Shegai, S. Schlücker, L.-L. Tay, K.G. Thomas, Z.-Q. Tian, R.P. Van Duyne, T. Vo-Dinh, Y. Wang, K.A. Willets, C. Xu, H. $\mathrm{Xu}, \mathrm{Y}$. Xu, Y.S. Yamamoto, B. Zhao, L.M. Liz-Marzán, Present and future of surfaceenhanced Raman scattering, ACS Nano 14 (1) (2020) 28-117, https://doi.org/ 10.1021/acsnano.9b04224.

[7] D.-Y. Wu, J.-F. Li, B. Ren, Z.-Q. Tian, Electrochemical surface-enhanced Raman spectroscopy of nanostructures, Chem. Soc. Rev. 37 (5) (2008) 1025, https://doi. org/10.1039/b707872m.

[8] S. Schlücker, Surface-enhanced Raman spectroscopy: concepts and chemical applications, Angew. Chemie Int. Ed. 53 (19) (2014) 4756-4795, https://doi. org/10.1002/anie.201205748.

[9] J.V. Perales-Rondon, A. Colina, M.C. González, A. Escarpa, Roughened silver microtubes for reproducible and quantitative SERS using a template-assisted electrosynthesis approach, Appl. Mater. Today. 20 (2020) 100710, https://doi. org/10.1016/j.apmt.2020.100710.

[10] J. Garoz-Ruiz, J.V. Perales-Rondon, A. Heras, A. Colina, Spectroelectrochemical sensing: current trends and challenges, Electroanalysis. 31 (7) (2019) 1254-1278, https://doi.org/10.1002/elan.v31.710.1002/elan.201900075.

[11] J.H.K. Pfisterer, F. Nattino, U.E. Zhumaev, M. Breiner, J.M. Feliu, N. Marzari, K.F. Domke, Role of $\mathrm{OH}$ intermediates during the au oxide electro-reduction at low $\mathrm{pH}$ elucidated by electrochemical surface-enhanced raman spectroscopy and implicit solvent density functional theory, ACS Catal. 10 (21) (2020) 12716-12726, https://doi.org/10.1021/acscatal.0c0275210.1021/acscatal.0c02752.s001.

[12] S. Hernandez, J.V. Perales-Rondon, A. Heras, A. Colina, Electrochemical SERS and SOERS in a single experiment: a new methodology for quantitative analysis, Electrochim. Acta. 334 (2020) 135561, https://doi.org/10.1016/j.electacta. 2019.135561.

[13] D. Martín-Yerga, A. Pérez-Junquera, M.B. González-García, J.V. Perales-Rondon, A. Heras, A. Colina, D. Hernández-Santos, P. Fanjul-Bolado, Quantitative Raman spectroelectrochemistry using silver screen-printed electrodes, Electrochim. Acta. 264 (2018) 183-190, https://doi.org/10.1016/j.electacta.2018.01.060.

[14] S. Hernandez, J.V. Perales-Rondon, A. Arnaiz, M. Perez-Estebanez, E. Gomez, A. Colina, A. Heras, Determination of nicotinamide in a multivitamin complex by electrochemical-surface enhanced Raman spectroscopy, J. Electroanal. Chem. 879 (2020) 114743, https://doi.org/10.1016/j.jelechem.2020.114743.

[15] D. Martín-Yerga, A. Pérez-Junquera, M.B. González-García, D. Hernández-Santos, P. Fanjul-Bolado, Towards single-molecule in situ electrochemical SERS detection with disposable substrates, Chem. Commun. 54 (45) (2018) 5748-5751, https:// doi.org/10.1039/C8CC02069H.

[16] D. Ibañez, E.C. Romero, A. Heras, A. Colina, Dynamic Raman Spectroelectrochemistry of Single Walled Carbon Nanotubes modified electrodes using a Langmuir-Schaefer method, Electrochim. Acta. 129 (2014) 171-176, https://doi. org/10.1016/j.electacta.2014.02.094.

[17] D. Ibañez, C. Fernandez-Blanco, A. Heras, A. Colina, Time-resolved study of the surface-enhanced Raman scattering effect of silver nanoparticles generated in voltammetry experiments, J. Phys. Chem. C. 118 (40) (2014) 23426-23433, https://doi.org/10.1021/jp5074363.

[18] D. Ibañez, D. Izquierdo, C. Fernandez-Blanco, A. Heras, A. Colina, Electrodeposition of silver nanoparticles in the presence of different complexing agents by time-resolved Raman spectroelectrochemistry, J. Raman Spectrosc. 49 (3) (2018) 482-492, https://doi.org/10.1002/jrs.v49.310.1002/jrs.5307.

[19] J.V. Perales-Rondon, S. Hernandez, D. Martin-Yerga, P. Fanjul-Bolado, A. Heras, A. Colina, Electrochemical surface oxidation enhanced Raman scattering, Electrochim. Acta 282 (2018) 377-383, https://doi.org/10.1016/j.electacta، 2018.06.079.

[20] S. Hernandez, J.V. Perales-Rondon, A. Heras, A. Colina, Determination of uric acid in synthetic urine by using electrochemical surface oxidation enhanced Raman scattering, Anal. Chim. Acta 1085 (2019) 61-67, https://doi.org/10.1016/j. aca.2019.07.057.

[21] L. Zhao, J. Blackburn, C.L. Brosseau, Quantitative detection of uric acid by electrochemical-surface enhanced Raman spectroscopy using a multilayered $\mathrm{Au}$ / Ag substrate, Anal. Chem. 87 (1) (2015) 441-447, https://doi.org/10.1021/ ac503967s.

[22] T.P. Lynk, C.S. Sit, C.L. Brosseau, Electrochemical surface-enhanced Raman spectroscopy as a platform for bacterial detection and identification, Anal. Chem. 90 (21) (2018) 12639-12646, https://doi.org/10.1021/acs.analchem. 8b0280610.1021/acs.analchem.8b02806.s001.

[23] R.A. Karaballi, A. Nel, S. Krishnan, J. Blackburn, C.L. Brosseau, Development of an electrochemical surface-enhanced Raman spectroscopy (EC-SERS) aptasensor for direct detection of DNA hybridization, Phys. Chem. Chem. Phys. 17 (33) (2015) 21356-21363, https://doi.org/10.1039/C4CP05077K.

[24] S.D. Bindesri, D.S. Alhatab, C.L. Brosseau, Development of an electrochemical surface-enhanced Raman spectroscopy (EC-SERS) fabric-based plasmonic sensor for point-of-care diagnostics, Analyst. 143 (17) (2018) 4128-4135, https://doi. org/10.1039/C8AN01117F.

[25] B. Sharma, R.R. Frontiera, A. Henry, E. Ringe, R.P. Van Duyne, SERS: Materials, applications, and the future Surface enhanced Raman spectroscopy (SERS) is a powerful vibrational, Mater. Today. 15 (2012) 16-25, https://doi.org/10.1016/ S1369-7021(12)70017-2.

[26] G.F.M. Ball, Water-soluble Vitamin Assays in Human Nutrition, Springer US, Boston, MA, 1994. doi: 10.1007/978-1-4615-2061-0.

[27] P. Nagaraja, R.A. Vasantha, H.S. Yathirajan, Spectrophotometric determination of folic acid in pharmaceutical preparations by coupling reactions with iminodibenzyl or 3-aminophenol or sodium molybdate-pyrocatechol, Anal. Biochem. 307 (2) (2002) 316-321, https://doi.org/10.1016/S0003-2697(02) 00038-6. 
[28] R. Matias, P.R.S. Ribeiro, M.C. Sarraguça, J.A. Lopes, A UV spectrophotometric method for the determination of folic acid in pharmaceutical tablets and dissolution tests, Anal. Methods 6 (9) (2014) 3065, https://doi.org/10.1039/ c3ay41874j.

[29] D.E. Breithaupt, Determination of folic acid by ion-pair RP-HPLC in vitaminfortified fruit juices after solid-phase extraction, Food Chem. 74 (4) (2001) 521-525, https://doi.org/10.1016/S0308-8146(01)00219-9.

[30] B.C. Nelson, K.E. Sharpless, L.C. Sander, Quantitative determination of folic acid in multivitamin/multielement tablets using liquid chromatography/tandem mass spectrometry, J. Chromatogr. A 1135 (2) (2006) 203-211, https://doi.org/ 10.1016/j.chroma.2006.09.040.

[31] M.J.A. Lima, G.P. Vieira, R.N. Fernandes, A.A. Tanaka, B.F. Reis, Development of a procedure based on chemiluminescence and multicommutation approach for the determination of folic acid in pharmaceuticals, J. Braz. Chem. Soc. 27 (2016) 153-160, https://doi.org/10.5935/0103-5053.20150265.

[32] A.A. Ensafi, H. Karimi-Maleh, Modified multiwall carbon nanotubes paste electrode as a sensor for simultaneous determination of 6-thioguanine and folic acid using ferrocenedicarboxylic acid as a mediator, J. Electroanal. Chem. 640 (12) (2010) 75-83, https://doi.org/10.1016/j.jelechem.2010.01.010.

[33] H. Beitollahi, M.M. Ardakani, B. Ganjipour, H. Naeimi, Novel 2,2'-[1,2ethanediylbis(nitriloethylidyne)]-bis-hydroquinone double-wall carbon nanotube paste electrode for simultaneous determination of epinephrine, uric acid and folic acid, Biosens. Bioelectron. 24 (3) (2008) 362-368, https://doi.org/10.1016/j. bios.2008.04.009.

[34] J.B. Raoof, N. Teymoori, M.A. Khalilzadeh, R. Ojani, A high sensitive electrochemical nanosensor for simultaneous determination of glutathione, NADH and folic acid, Mater. Sci. Eng. C 47 (2015) 77-84, https://doi.org/ 10.1016/j.msec.2014.11.012.

[35] D. Manoj, D. Ranjith Kumar, J. Santhanalakshmi, Impact of $\mathrm{CuO}$ nanoleaves on MWCNTs/GCE nanocomposite film modified electrode for the electrochemical oxidation of folic acid, Appl. Nanosci. 2 (3) (2012) 223-230, https://doi.org/ 10.1007/s13204-012-0093-9.

[36] Y.C. Lu, W.H. Chiang, C.Y. Liu, J.P. Chu, H.C. Ho, C.H. Hsueh, Wafer-scale SERS metallic nanotube arrays with highly ordered periodicity, Sensors Actuators, B Chem. 329 (2021) 1-7, https://doi.org/10.1016/j.snb.2020.129132.

[37] Y.i. Li, L.u. Yu, J. Li, L. Wang, R. Lu, Polyamide@Ag coralloid nanoarrays with 3D high-density hot spots for ultrasensitive SERS sensing, Chem. Eng. J. 397 (2020) 125434, https://doi.org/10.1016/j.cej.2020.125434.

[38] J.J. Castillo, T. Rindzevicius, C.E. Rozo, A. Boisen, Adsorption and vibrational study of folic acid on gold nanopillar structures using surface-enhanced Raman scattering spectroscopy, Nanomater. Nanotechnol. 5 (2015) 29, https://doi.org/ $10.5772 / 61606$.

[39] W. Ren, Y. Fang, E. Wang, A binary functional substrate for enrichment and ultrasensitive SERS spectroscopic detection of folic acid using graphene oxide/Ag nanoparticle hybrids, ACS Nano. 5 (8) (2011) 6425-6433, https://doi.org/ $10.1021 / \mathrm{nn} 201606 \mathrm{r}$.

[40] M. Perez-Estebanez, S. Hernandez, J.V. Perales-Rondon, E. Gomez, A. Heras, A. Colina, Chemical selectivity in electrochemical surface oxidation enhanced Raman scattering, Electrochim. Acta 353 (2020) 136560, https://doi.org/10.1016/ j.electacta.2020.136560.

[41] R.A.R. Teixeira, F.R.A. Lima, P.C. Silva, L.A.S. Costa, A.C. Sant'Ana, Tracking chemical interactions of folic acid on gold surface by SERS spectroscopy, Spectrochim. Acta Part A Mol. Biomol. Spectrosc. 223 (2019) 117305, https:// doi.org/10.1016/j.saa.2019.117305.

[42] J. Mocak, A.M. Bond, S. Mitchell, G. Scollary, A statistical overview of standard (IUPAC and ACS) and new procedures for determining the limits of detection and quantification: application to voltammetric and stripping techniques (Technical Report), Pure Appl. Chem. 69 (1997) 297-328, https://doi.org/10.1351/ pac199769020297. 